\title{
Extensive bone metastases as the initial symptom of papillary thyroid microcarcinoma: A case report
}

\author{
WEI ZHENG, JIAN TAN and GUIZHI ZHANG \\ Department of Nuclear Medicine, Tianjin Medical University General Hospital, Tianjin 300052, P.R. China
}

Received August 12, 2014; Accepted March 25, 2015

DOI: $10.3892 / \mathrm{etm} .2015 .2423$

\begin{abstract}
A 53-year-old female patient presented with lumbar and pelvic pain. The ${ }^{18} \mathrm{~F}$-fluorodeoxyglucose ( $\left.{ }^{18} \mathrm{~F}-\mathrm{FDG}\right)$ positron emission tomography/computed tomography scintigraphy showed extensive bone destruction with hypermetabolic osteolysis, with increased ${ }^{18} \mathrm{~F}-\mathrm{FDG}$ uptake. The histological examination of the pubic bone lesion revealed a metastatic tumor. Neither chemotherapy nor radiotherapy could control the condition. More than a year later, the patient underwent a total thyroidectomy with cervical lymph node clearance. The histological examination revealed a papillary thyroid microcarcinoma (PTMC) in the left lobe with capsular invasion. ${ }^{131}$ I treatment with thyroid hormone withdrawal followed. The ${ }^{131}$ I whole-body scintigraphy showed extensive uptake in the basic thyroid deposits, most bones and both lungs. Three months after the ${ }^{131}$ I treatment, the patient succumbed to cachexia. The present case indicated that not all PTMCs have excellent prognosis and that, despite being rare, clinically significant metastases can arise from a PTMC.
\end{abstract}

\section{Introduction}

Papillary thyroid microcarcinomas (PTMCs) comprise a special subgroup of papillary thyroid cancer (PTC). According to the World Health Organization, PTMCs have a maximum diameter of $\leq 10 \mathrm{~mm}$ (1) and may account for up to $30 \%$ of all PTCs $(2,3)$. Despite the fact that PTMCs were previously considered to be benign neoplasms with a long and indolent course, certain studies have indicated that PTMCs are frequently detected in patients exhibiting poor prognostic factors, such as bilaterality, extrathyroid extension, multifocality and lymph node or distant metastasis (4-6). The present

Correspondence to: Professor Jian Tan, Department of Nuclear Medicine, Tianjin Medical University General Hospital, 154 Anshan Road, Heping, Tianjin 300052, P.R. China

E-mail: tanpost@163.com

Key words: papillary thyroid carcinoma, microcarcinoma, extensive bone metastases, ${ }^{131}$ I whole body scintigraphy, PET/CT report describes the case of a patient with PTMC whose initial symptom was extensive bone metastases. The patient succumbed to cachexia after two years, having received surgical therapy, chemotherapy, radiotherapy and a single ${ }^{131} \mathrm{I}$ treatment, an infrequent clinical outcome. The present case study emphasizes that not all PTMCs are associated with a good prognosis, and therefore, further studies of PTMCs are required.

\section{Case report}

In September 2011, a 53-year-old female patient, who had no significant medical history, presented with osteodynia in the lumbar region and pelvic area that lasted several months. The ${ }^{18} \mathrm{~F}$-fluorodeoxyglucose positron emission tomography/computed tomography $\left({ }^{18} \mathrm{~F}-\mathrm{FDG}\right.$ PET/CT) scintigraphy showed that there was extensive bone destruction with hypermetabolic osteolysis in the skull, spine, sternum, certain ribs, bilateral scapulas, ilia, pubis, ischia and left femur, which had increased ${ }^{18}$ F-FDG uptake (Fig. 1). The PET scintigraphy showed that the right lobe of the thyroid had patchy increased ${ }^{18}$ F-FDG uptake, which was highly suspicious of malignancy. The patient underwent pubic bone lesion resection 10 days later. The histological examination confirmed the diagnosis of a metastatic tumor (Fig. 2).

Following diagnosis, the patient received six courses of taxotere and paraplatin chemotherapy and a pamidronate disodium treatment course the following year. At the same time as the pamidronate disodium treatment, three-dimensional conformal radiotherapy (planning target volume $30 \mathrm{~Gy} / 10 \mathrm{f}$ ) was performed on the left side of the femoral head; left acetabulum; sternum; the 5th, 11th and 12th thoracic vertebrae; and the 1st and 4th lumbar vertebrae, 45 times in total. Another ${ }^{18} \mathrm{~F}$-FDG PET/CT scintigraphy that was performed in January 2013 (Fig. 3) showed more severe bone destruction as well as a notably increased ${ }^{18} \mathrm{~F}$-FDG uptake in most of the previous bone lesions and within multiple pulmonary nodules and lymph nodes, which led the doctors to consider the possibility of metastatic tumors. The patient then underwent a total thyroidectomy with central and laterocervical lymph node clearance on the right side. The histological examination of the surgical specimen identified a PTMC with follicular architecture of $6 \mathrm{~mm}$ in the left lobe with capsular invasion and nodular goiter, and adenomatous hyperplasia in the right lobe (Fig. 4). The PTMC was in the left lobe, although the 


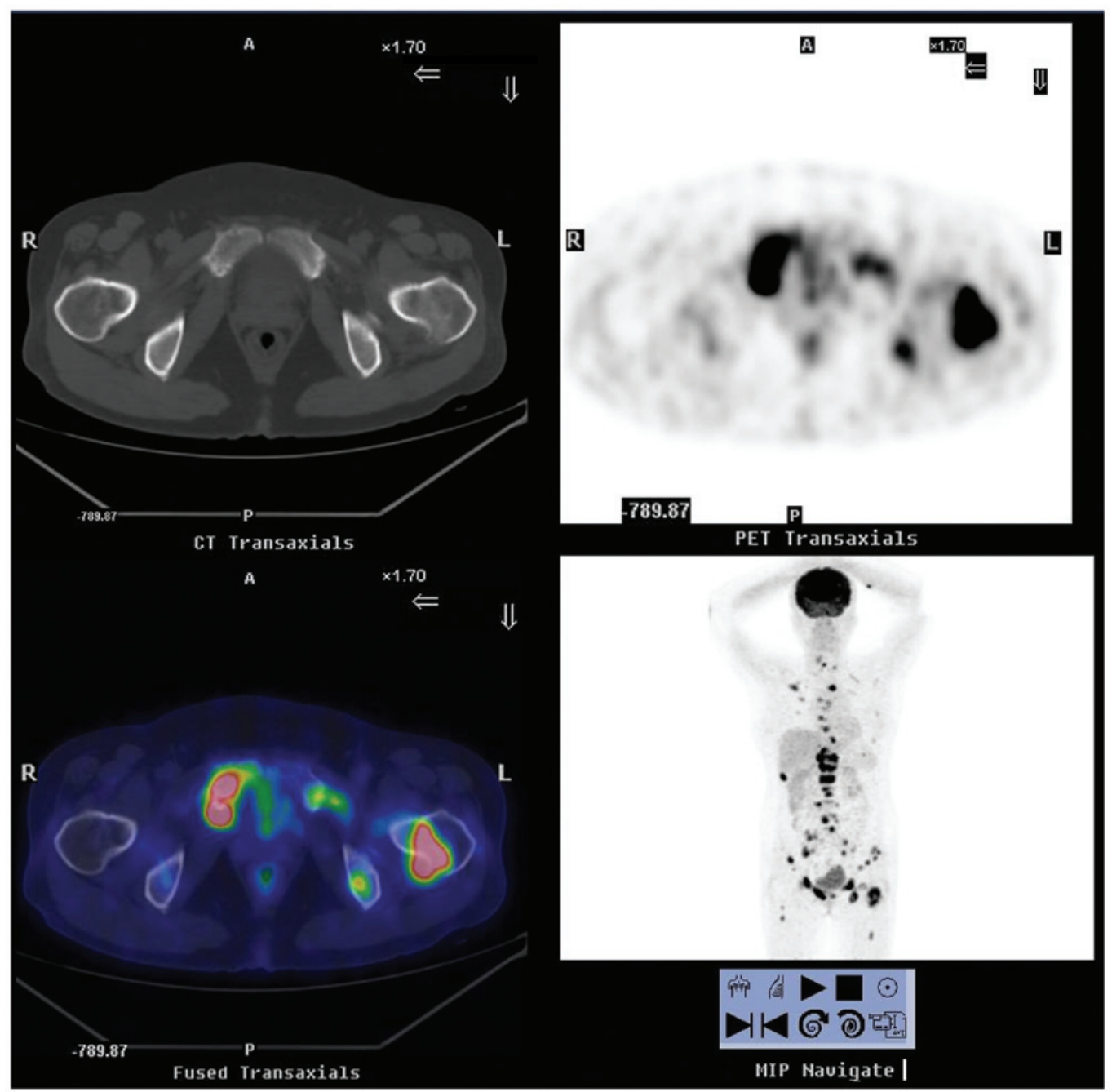

Figure 1. ${ }^{18} \mathrm{~F}$-FDG PET/CT scintigraphy from September 2011, showing extensive bone destruction with hypermetabolic osteolysis in the skull, spine, sternum, certain ribs, bilateral scapulas, ilia, pubis, ischia and left femur, which had increased ${ }^{18} \mathrm{~F}$-FDG uptake. ${ }^{18} \mathrm{~F}$-FDG PET/CT, ${ }^{18} \mathrm{~F}$-fluorodeoxyglucose positron emission tomography/computed tomography; A, anterior; P, posterior; R, right; L, left.

A

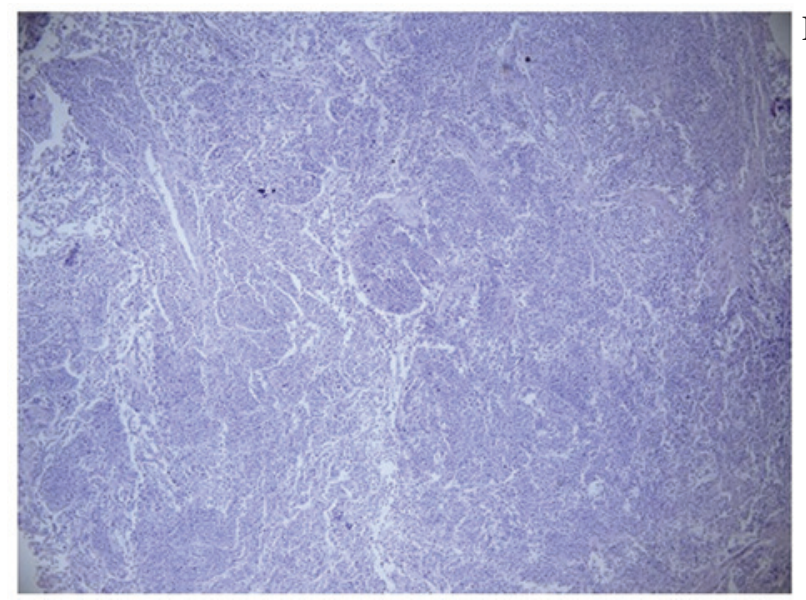

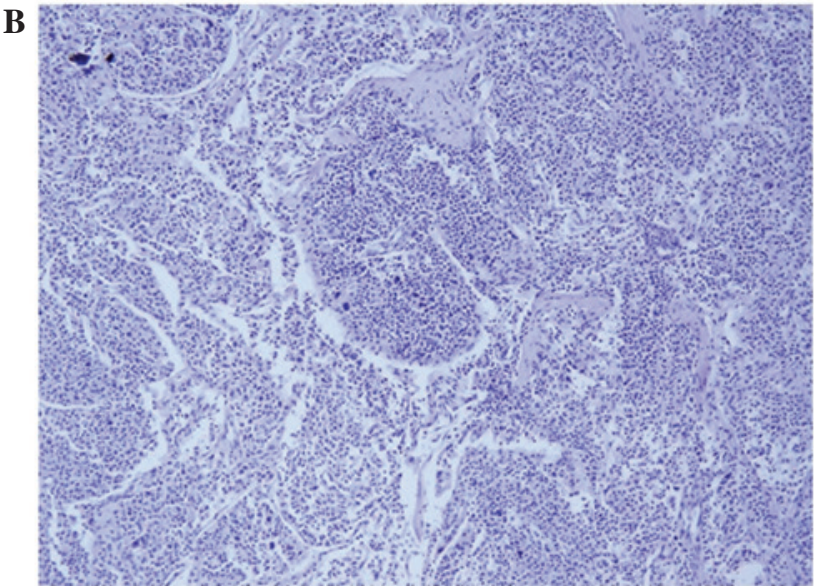

Figure 2. Histological examination of the pubic bone lesion revealed a metastatic tumor. The tissue was stained with hematoxylin and eosin. (A) Magnification, $\mathrm{x} 40$; (B) magnification, $\mathrm{x} 100$.

first PET/CT scintigraphy indicated that the right lobe of thyroid exhibited increased ${ }^{18} \mathrm{~F}$-FDG uptake, which was highly suggestive of malignancy. There was no cervical lymphoid involvement.
Following the surgery, the patient was given levothyroxine sodium (50 $\mu \mathrm{g} / \mathrm{day})$ and morphine sulfate for analgesia. Two months later, due to an accidental fall, the patient's physical activity was limited as a result of coccyx pain. In April, 


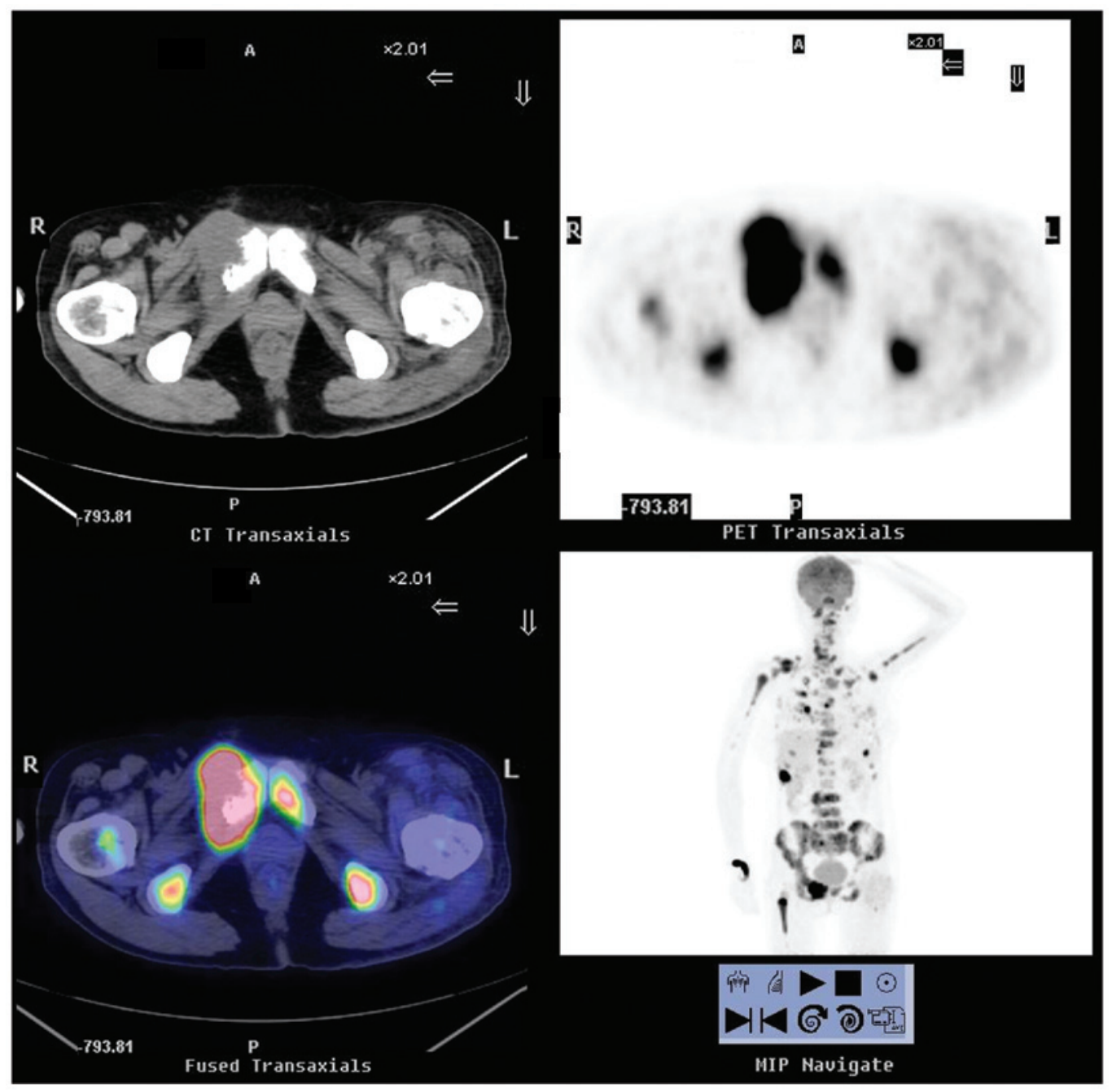

Figure 3. ${ }^{18} \mathrm{~F}$-FDG PET/CT scintigraphy from January 2013, showing more severe bone destruction as well as a notably increased ${ }^{18} \mathrm{~F}-\mathrm{FDG}$ uptake in most of the previous bone lesions and within multiple pulmonary nodules and lymph nodes. This pointed to the presence of metastatic tumors. ${ }^{18} \mathrm{~F}-\mathrm{FDG}$ PET/CT, ${ }^{18} \mathrm{~F}$-fluorodeoxyglucose positron emission tomography/computed tomography; A, anterior; P, posterior; R, right; L, left.

A

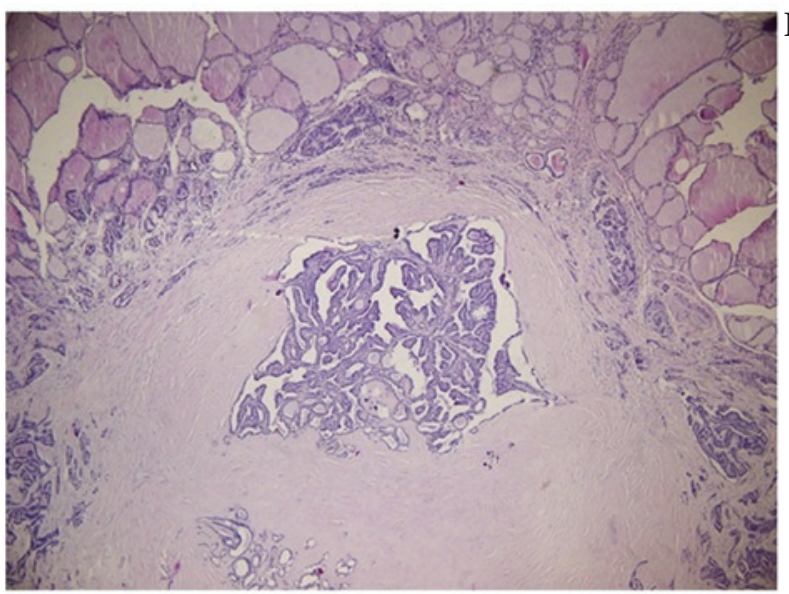

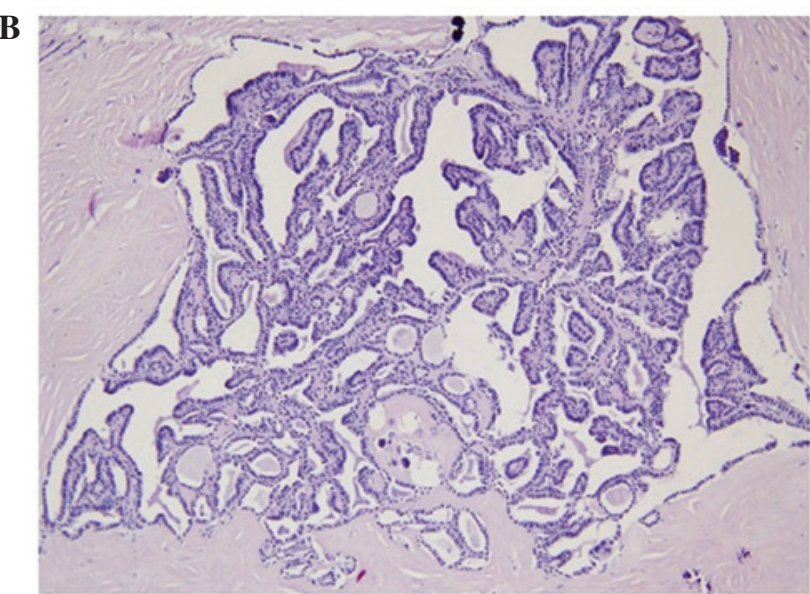

Figure 4. Histological examination of the surgical specimen revealed a papillary thyroid microcarcinoma with follicular architecture of $6 \mathrm{~mm}$ in the left lobe with capsular invasion. The tissue was stained with hematoxylin and eosin. (A) Magnification, x40; (B) magnification, x100.

radioiodine treatment with thyroid hormone withdrawal (THW) was carried out $\left(3.7 \mathrm{GBq}{ }^{131} \mathrm{I}\right)$. The subsequent post-therapeutic whole-body scintigraphy showed extensive uptake in the basic thyroid deposits, most bones and both lungs (Fig. 5).
Three months after the treatment, the patient succumbed to cachexia. The thyroglobulin ( $\mathrm{Tg}$ ) level had always remained $>300(0-55) \mathrm{ng} / \mathrm{ml}$, from the presurgical stage to during and following THW. The Tg antibody level was normal. 


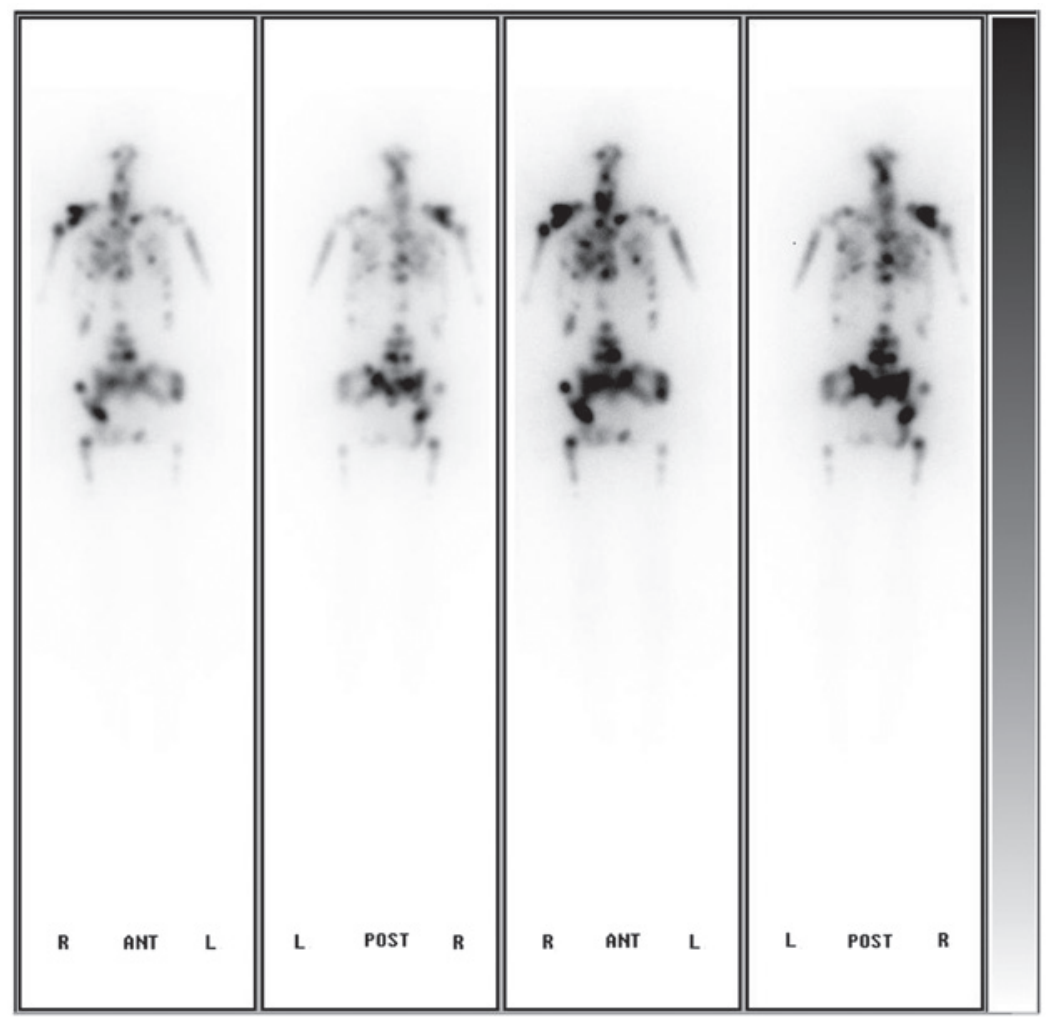

Figure 5. Subsequent post-therapeutic whole body scintigraphy showing extensive uptake of ${ }^{131} \mathrm{I}$ in basic thyroid deposits, most bones and both lungs. A, anterior; $\mathrm{P}$, posterior.

\section{Discussion}

PTMCs are tumors measuring $\leq 10 \mathrm{~mm}$ in diameter. These tumors are undetectable at clinical examination and are often found incidentally following thyroidectomy performed for other reasons. Due to the increasing use of cervical ultrasound and fine needle aspiration, the rate of diagnosis of PTMCs is constantly rising, but the majority of these tumors are associated with an excellent prognosis and their course is long and indolent (7). In certain cases, however, PTMCs can result in recurrence, distant metastasis and even mortality (8).

Histological and clinical risk factors that are associated with malignant behavior have been identified in the medical literature and include the following: Age of patient, $>45$ years; tumor size, $>5 \mathrm{~mm}$; vascular invasion; multifocal carcinoma; extrathyroid extension; node involvement and $\mathrm{BRAF}^{\mathrm{V} 600 \mathrm{E}}$ mutations (9-11). In the present case, despite the gene test not being performed, the patient still exhibited almost half of the aforementioned poor prognostic factors, including the age, tumor size and vascular invasion.

In the medical literature, only a few cases of distant metastatic PTMCs have been reported. Mercante et al (6) described four metastatic cases in a series of 445 patients with PTMC. All those metastases had extended outside the thyroid or showed lymph node involvement. The present case illustrates how clinically significant distant metastases, including extensive bone or lung metastases, can arise from PTMC. The fact that the extensive bone metastases constituted the initial symptom is particularly noteworthy. The metastases imposed severe bone pain to the patient, which could not be controlled by either chemotherapy or radiotherapy, and neither surgery nor radioiodine therapy could relieve the condition. The patient succumbed 22 months later.

Kim et al (13) proposed that PTMC should no longer be considered to be a simple occult indolent thyroid cancer, but as an early stage of a disease that will, eventually, develop into PTC, since the gene expression profiles of PTMCs are not different from those of PTCs. Due to the differential definitions of PTMC, however, the therapeutic approaches for the condition have not yet been standardized, and range from more conservative to more aggressive treatment $(6,14,15)$; therefore, the management of PTMC has become a controversial issue. Although PTMC is generally associated with an excellent prognosis, it has a mortality rate of $0.5 \%$ (16). The presence of at least two of the risk factors stated below is strongly associated with cancer-related mortality and can help to identify candidates for more aggressive treatment (7). The risk factors that point towards the necessity of a more aggressive therapeutic approach include unfavorable histology, capsule infiltration, multifocality and evidence of locoregional or distant metastasis. Certain studies have recommended radioiodine ablation and total thyroidectomy for high-risk patients with PTMC, on the basis that this would reduce the recurrence rate of the disease and facilitate the use of serum $\mathrm{Tg}$ for postsurgical follow-up $(17,18)$.

In conclusion, the present case report has emphasized that PTMC can lead to clinically significant metastases. The report has described an extremely rare case in which extensive bone metastases were the initial symptom of PTMC. The case highlights that not all PTMCs are associated with a good prognosis and, therefore, further research on PTMCs is required. 


\section{References}

1. LiVolsi VA, Albores-Saavedra J, Asa SL, et al: Papillary Carcinoma In: Pathology and Genetics of Tumours of Endocrine Organs. World Health Organization Classification of Tumours. DeLellis RA Lloyd RV, Heitz PU and Eng C (eds). Vol. 8. Third edition. IARC Press, Lyon, pp57-66, 2004

2. Ito Y, Tomoda $\mathrm{C}$, Uruno $\mathrm{T}$, et al: Papillary microcarcinoma of the thyroid: How should it be treated? World J Surg 28: 1115-1121, 2004

3. Sorrentino F, Atzeni J, Romano G, Buscemi G and Romano M: Differentiated microcarcinoma of the thyroid gland. G Chir 31: 277-278, 2010 (In Italian).

4. Cho JK, Kim JY, Jeong CY, Jung EJ, Park ST, Jeong SH, et al: Clinical features and prognostic factors in papillary thyroid microcarcinoma depends on age. J Korean Surg Soc 82: 281-287, 2012.

5. Page C, Biet A, Boute P, Cuvelier P and Strunski V: 'Aggressive papillary' thyroid microcarcinoma. Eur Arch Otorhinolaryngol 266: 1959-1963, 2009.

6. Mercante G, Frasoldati A, Pedroni C, et al: Prognostic factors affecting neck lymph node recurrence and distant metastasis in papillary microcarcinoma of the thyroid: Results of a study in 445 patients. Thyroid 19: 707-716, 2009.

7. Yu XM, Wan Y, Sippel RS and Chen H: Should all papillary thyroid microcarcinomas be aggressively treated? An analysis of 18,445 cases. Ann Surg 254: 653-660, 2011.

8. Cappelli C, Castellano M, Braga M, et al: Aggressiveness and outcome of papillary thyroid carcinoma (PTC) versus microcarcinoma (PMC): A mono-institutional experience. J Surg Oncol 95: 555-560, 2007.

9. Basolo F, Torregrossa L, Giannini R, et al: Correlation between the BRAF V600E mutation and tumor invasiveness in papillary thyroid carcinomas smaller than 20 millimeters: Analysis of 1060 cases. J Clin Endocrinol Metab 95: 4197-4205, 2010.
10. Lee KJ, Cho YJ, Kim SJ, et al: Analysis of the clinicopathologic features of papillary thyroid microcarcinoma based on 7-mm tumor size. World J Surg 35: 318-323, 2011.

11. Vasileiadis I, Karakostas E, Charitoudis G, et al: Papillary thyroid microcarcinoma: Clinicopathological characteristics and implications for treatment in 276 patients. Eur J Clin Invest 42: 657-664, 2012

12. Zhang L, Wei WJ, Ji QH, Zhu YX, Wang ZY, Wang Y, et al: Risk factors for neck nodal metastasis in papillary thyroid microcarcinoma: A study of 1066 patients. J Clin Endocrinol Metab 97: 1250-1257, 2012.

13. Kim HY, Park WY, Lee KE, Park WS, Chung YS, Cho SJ and Youn YK: Comparative analysis of gene expression profiles of papillary thyroid microcarcinoma and papillary thyroid carcinoma. J Cancer Res Ther 6: 452-457, 2010.

14. Arora N, Turbendian HK, Kato MA, Moo TA, Zarnegar R and Fahey TJ III : Papillary thyroid carcinoma and microcarcinoma: Is there a need to distinguish the two? Thyroid 19: 473-477, 2009.

15. Orsenigo E, Beretta E, Fiacco E, Scaltrini F, Veronesi P, Invernizzi L, et al: Management of papillary microcarcinoma of the thyroid gland. Eur J Surg Oncol 30: 1104-1106, 2004.

16. Yu XM, Wan Y, Sippel RS and Chen H: Should all papillary thyroid microcarcinomas be aggressively treated? An analysis of 18,445 cases. Ann Surg 254: 653-660, 2011

17. Dietlein M, Luyken WA, Schicha $\mathrm{H}$ and Larena-Avellaneda A: Incidental multifocal papillary microcarcinomas of the thyroid: Is subtotal thyroidectomy combined with radioiodine ablation enough? Nucl Med Commun 26: 3-8, 2005.

18. Sakorafas GH, Giotakis J and Stafyla V: Papillary thyroid microcarcinoma: A surgical perspective. Cancer Treat Rev 31: 423-438, 2005. 